\title{
A Nomogram to Predict Breast Tumor Regression Patterns after Neoadjuvant Chemotherapy Based on Baseline Characteristics
}

\section{Xiaosheng Zhuang}

Guangdong General Hospital

\section{Minyi Cheng}

Guangdong General Hospital

\section{Liulu Zhang}

Guangdong General Hospital

Fei Ji

Guangdong General Hospital

\section{Teng Zhu}

Guangdong General Hospital

\section{Ciqiu Yang}

Guangdong General Hospital

\section{Mei Yang}

Guangdong General Hospital

Hongfei Gao

Guangdong General Hospital

Jieqing Li

Guangdong General Hospital

Kun Wang ( $\nabla$ gzwangkun@126.com )

Guangdong General Hospital https://orcid.org/0000-0001-9851-7080

\section{Research}

Keywords: Breast Cancer, Chemotherapy, Breast Conserving Surgery, Tumor Regression

Posted Date: August 14th, 2020

DOI: https://doi.org/10.21203/rs.3.rs-57234/v1

License: (c) (1) This work is licensed under a Creative Commons Attribution 4.0 International License.

Read Full License 


\section{Abstract}

Background Breast cancers show different regression patterns after neoadjuvant chemotherapy. Certain regression patterns are associated with more reliable margins in breast-conserving surgery. Our study aims to establish a nomogram based on clinicopathological factors and laboratory indicators to predict regression patterns in breast cancer patients.

Methods We retrospectively reviewed 320 patients with stage II-III breast cancer who received neoadjuvant chemotherapy and had definitive surgery in our center from January 2016 to December 2019. Tumor regression patterns were categorized as type 1 (concentric regression $+p C R$ ) and type 2 (multifocal residues + SD + PD) based on mass pathological results. A multivariate logistic regression model was applied to distinguish predictive factors for tumor regression patterns. A nomogram was built based on five predictive factors from the multivariate logistic regression model.

Results Molecular subtypes were not significantly associated with tumor regression patterns. Multivariate analysis identified five independent indicators: menopausal status, estrogen receptor status, T stage, $\mathrm{N}$ stage and lymphocyte to monocyte ratio. The nomogram yielded an area under the curve (AUC) of 0.70 (95\% confidence interval $0.62-0.78$ ) based on these factors. The calibration plot accompanied by the Hosmer-Lemeshow goodness-of-fit (GOF) test ( $p$-value $=0.9$ ) showed good consistency between the estimated probabilities and the actual rate of type 1 cases.

Conclusion HR+/HER2- breast cancers are more likely to have type 2 regression after neoadjuvant chemotherapy. Five baseline factors, including clinicopathological factors and laboratory indexes, were incorporated to establish a nomogram, which exhibited a satisfactory discriminatory ability for predicting different patterns of tumor regression.

\section{Introduction}

Preoperative neoadjuvant chemotherapy (NAC) is an increasingly accepted treatment option for patients with locally advanced breast cancers[1]. It enables the downstaging of breast cancers and improves the possibility of breast-conserving therapy in most breast cancers[2,3]. Primary tumors shrink after NAC and show various regression patterns, including pathologic complete response (pCR), concentric shrinkage, multiple residual foci, main residual disease with satellite foci, stable disease (SD) and progressive disease (PD)[4-6]. Among these patterns, unifocal residual disease and PCR favor the adoption of breastconserving surgery (BCS). However, patients with multiple residual foci, SD and PD are more prone to receiving mastectomy after completing NAC.

The local recurrence rate in NAC was found to be significantly higher than that in adjuvant chemotherapy. A meta-analysis of 4756 patients with breast cancer revealed that the difference in the 15-year local recurrence rates between the preoperative and postoperative chemotherapy groups was $5.5 \%$ ( $21.4 \%$ vs $15.9 \%, p=0.0001)[7]$. The finding was proposed to be caused by an increase in BCS in favorable responders to NAC who would otherwise have undergone mastectomy. In cancers with multifocal 
regression, negative margins in a BCS might not be reliable, which is due to the micro/satellite foci remaining. In their study, a higher ipsilateral breast tumor recurrence rate (IBTR) did not cause a significant difference in overall survival. However, it brought an extra operation and may cause mental burden to patients. Recently, researchers from the University of Pittsburgh showed that multifocal regression is an independent predictive factor for IBTR[6]. A total of $9.5 \%$ of patients underwent a second operation due to positive margins in their first attempted BCS.

Patients showing poor response to NAC, including SD and PD, are obviously less likely to undergo BCS. Based on clinical interest, we categorized tumor regression patterns into 2 types. The type 1 regression pattern consists of pCR and unifocal regression, which are favorable candidates for BCS. The type 2 regression pattern includes multiple residual foci, main residual disease with satellite foci, stable disease (SD) and progressive disease (PD), for which a mastectomy may be more appropriate. Many researchers are interested in predicting the $\mathrm{PCR}$ to $\mathrm{NAC}[8,9]$, but few have focused on predicting tumor regression patterns, which mainly concern the safety and feasibility of BCS. Apart from patients' willingness and contraindications to radiation therapy[10], the tumor regression pattern is essential for determining whether BCS is feasible for an individual patient. Therefore, the early prediction of the tumor regression pattern is of great significance and needs to be explored.

The prediction of tumor regression at baseline enables the early identification of candidates for BCS. For those predicted to have type 2 regression with great possibility, treatment strategies may be modified at the early stage to achieve a better response to NAC. For instance, in triple-negative breast cancers, a more vigorous platinum-based chemotherapy regimen may be considered in non-BCS candidates[11]. In this original article, a nomogram was established to predict type 1 regression based on baseline clinicopathological factors and laboratory indexes.

\section{Patients And Methods}

\section{Patients and factors}

This study was approved by the Ethics Committee of our hospital (No.GDREC2019764H) and was conducted in accordance with the principles of the Declaration of Helsinki. The requirement for informed consent was waived with authorization because our study had a retrospective design and no additional interventions were involved. In our clinical practice, patients who have luminal breast cancer and desire breast conservation but have large tumor relative to their breast size or those with positive axillary nodes are selected as candidates for neoadjuvant chemotherapy. We retrospectively enrolled patients with stage II-III breast cancers who both received NAC and underwent surgery in Guangdong General Hospital between February 2016 and December 2019. The inclusion criteria were as follows: 1) biopsy-confirmed invasive breast cancer with no distant metastasis; 2) no missing baseline clinicopathological factors or laboratory indexes; and 3) definitive surgery following standard preoperative chemotherapy. The exclusion criteria were as follows: 1) lack of baseline characteristics and laboratory results; 2) patients who underwent final surgery at an external cancer center and the pathologic result was not available; and 
3) patients who did not complete the planned NAC regimen or had nonstandard NAC treatment (e.g., trastuzumab was not included in the presurgical chemotherapy). All patients in our center underwent MRI at baseline to determine the extent and focality of the primary breast cancer.

In this retrospective study, each enrolled patient underwent the planned standard chemotherapy regimen as recommended by the NCCN Breast Cancer Guideline[10]. The preoperative chemotherapy regimens suggested by qualified oncologists were taxane-based, anthracycline-based or a combination of the two. Furthermore, for patients with HER-2 positive status, anti-HER-2 therapy (e.g., trastuzumab) was added in the treatment.

\section{Immunohistochemistry $(\mathrm{IHC})$}

IHC was performed for each patient to determine the baseline estrogen receptor (ER) status, progesterone receptor (PR) status, HER-2 status, and Ki67 status. The cutoff value for ER and PR was set at 1\%, and the cutoff value for Ki67 was $20 \%$. In regard to HER-2 status, tumors with an IHC staining of 0 to $1+$ were defined as HER-2 negative and those with 3+ as HER-2 positive. For tumors with an IHC staining of 2+, ISH testing was further performed to confirm the HER-2 status. A non-amplified ISH result denotes the HER-2 status as negative, and an amplified result denotes the HER-2 status as positive.

\section{Determination of tumor regression patterns}

The tumor regression patterns were determined based on the final surgical specimen. The patients underwent either BCS or mastectomy after completing NAC. In either surgical procedure, the operation zone covered the area of the primary cancer. Standard pathologic analyses were performed for the assessment of the residual tumor after preoperative chemotherapy. Surgical specimens were fixed with standard formalin solution by experienced breast pathologists and processed in standard breast tissue processors, after which the specimens were cut in parallel in a continuous manner to expose the cancer tissue. For each slice, a $5 \mathrm{~mm}$ slide was further prepared for H\&E staining. The longest diameter of the residual tumor was recorded. Type 1 regression included PCR and unifocal regression. The former was defined as no residual invasive cancer remaining after NAC (ypTis allowed)[12]. The latter was designated unifocal residual cancer tissue when the area of the original cancer with the longest diameter shrank at least $30 \%$. Type 2 regression comprised multiple residual foci, main residual disease with satellite foci, stable disease (SD) and progressive disease (PD). Multiple residual foci showed at least 2 separate foci in the continuous slides, whereas main residual disease with satellite foci showed a dominant residual disease with at least 1 minor accompanying focus. SD and PD were defined according to RECIST Version 1.1. PD indicated an increase of $\geq 20 \%$ in the largest diameter of the tumor. SD indicated an increase of less than $20 \%$ or a decrease of less than $30 \%$ in the tumor's largest diameter. These definitions were applied to both unifocal and multifocal breast cancers at baseline. The remaining tumor foci (if any) after preoperative systemic therapy were analyzed and categorized based on the same definitions. Three breast pathologists with more than 15 years of experience in breast pathology independently examined the specimens and determined the tumor regression pattern based on the H\&E- 
stained slides. Agreements were made on the tumor regression patterns among 3 pathologists to reduce possible errors caused by interobserver variability.

\section{Statistical analysis}

Among all the clinicopathological factors and laboratory indicators, 4 variables were treated as continuous variables, including age at diagnosis, body mass index (BMI), fibrinogen level, and D-dimer. Clinical factors, IHC indicators, lymphocyte to monocyte ratio (LMR) and platelet to lymphocyte ratio (PLR) were analyzed as categorical factors. For the two hematological factors, cutoff values were set when the Youden indexes were maximized. For assessing the correlations between tumor regression patterns and the listed variables, Mann-Whitney $U$ tests or chi-square tests were used where appropriate. We used logistic regression to perform univariate analyses on each factor. Those factors with a $p$-value $<0.1$ were incorporated into the multivariate analysis. Stepwise selection following Akaike's information criterion was performed to distinguish the optimal predictive elements for tumor regression patterns. We performed ROC analysis using the package " $\mathrm{pROC}$ " in R software. Five independent predictive factors identified in the multivariate logistic regression were included in the nomogram. To further assess the ability of our nomogram, a calibration plot and a decision curve were employed. The Hosmer-Lemeshow goodness-of-fit (GOF) test was applied to evaluate the concordance between the estimated probability and the actual rate of type 1 cases. A p-value $>0.05$ showed a satisfactory fit of the model. Decision curve analysis was also implemented to assess the model's usefulness in predicting tumor regression patterns. The nomogram and decision curve analysis were performed using the "rms" and "rmda" packages in $\mathrm{R}$ software, respectively.

\section{Results}

From January 2016 to December 2019, a total of 320 eligible patients who met the inclusion criteria were retrospectively enrolled in our cancer center. Table 1 shows the patients' clinicopathological factors and laboratory indexes. The median age in our cohort was 49 years (95\% range 33-65), and the median BMI was $23.5 \mathrm{~kg} / \mathrm{m} 2$ (95\% range 18.0-29.0). The median levels were $3.43 \mathrm{~g} / \mathrm{L}$ (95\% range 2.00-5.00) for fibrinogen and $405 \mathrm{ng} / \mathrm{ml}$ (95\% range 220-1016) for D-dimer. A total of 224 (70.0\%) patients were premenopausal, and 96 (30.0\%) were postmenopausal. The optimal cut-off values for the platelet to lymphocyte ratio (PLR) and lymphocyte to monocyte ratio (LMR) were 132 and 5.32, respectively. The cutoff values were $1 \%$ for both estrogen receptor (ER) status and progesterone receptor (PR) status and $20 \%$ for Ki67. A total of 145 (45.3\%) patients were identified as HER2-positive, and 175 (54.7\%) were HER2-negative. According to the latest TNM staging system, among the 320 cases enrolled, 260 (81.3\%) were classified as cT2, 60 (18.7) were classified as cT3-4, 136 (42.5\%) were classified as cN0 and 184 (57.5\%) were classified as $\mathrm{cN}+$. Additionally, 237 (74.1\%) patients had unifocal disease at baseline, and 83 (25.9\%) had multifocal disease. Among our patients, 111 (34.7\%) had hormone receptorpositive/HER2-negative (HR+/HER2-) breast cancers, 77 (24.1\%) had HR+/HER2+ breast cancers, 70 (21.8\%) had HR-/HER2+ (equal to HER2 overexpression) breast cancers and 62 (19.4\%) had HR-/HER2(equal to triple-negative) breast cancers. HR+/HER2- breast cancers had a significantly higher rate of type 
2 regression than the other subtypes $(24.3 \%$ vs $12.4 \%, p=0.006)$. No significant difference was found in the proportion of type 2 regression between HR-/HER2+ and HR-/HER2- breast cancers $(11.4 \%$ vs $12.9 \%$, $p=0.067)$. In our cohort, 267 (83.4\%) cases showed type 1 regression, and 53 (16.6\%) showed type 2 regression after NAC. The two regression patterns are shown in Figure 1.

The associations between the tumor regression patterns and clinicopathological factors are demonstrated in Table 1. The tumor regression patterns were significantly associated with BMI, LMR, menopausal status, T stage and $\mathrm{N}$ stage $(p \leq 0.05)$, and the borderline regression patterns were significantly associated with D-dimer level $(p=0.056)$ and ER status $(p=0.051)$. Table 2 lists the results of the univariate and multivariate logistic analyses. Patients who were premenopausal or ER-negative and had high LMR and later T/N stages had a higher rate of type 1 tumor regression. Additionally, the multivariate logistic regression analysis identified menopausal status, ER status, $\mathrm{T}$ stage, $\mathrm{N}$ stage and LMR as independent factors for predicting the type 1 tumor regression pattern after NAC.

Table 1. Clinical features of 320 patients and their correlations with regression patterns 


\begin{tabular}{|c|c|c|c|c|}
\hline 's & Total & Type 1 regression & Type 2 regression & $p$ value \\
\hline зars [median (5-95\%)] & $49(33-65)$ & $49(32.4-65.0)$ & $52(32.5-65.0)$ & 0.072 \\
\hline $\mathrm{g} / \mathrm{m} 2$ [median (5-95\%)] & $23.5(18.0-29.0)$ & $23.0(18.0-29.0)$ & $24.0(21.0-29.0)$ & 0.026 \\
\hline gen [median (5-95\%)] & $3.43(2.00-5.00)$ & $3.00(2.00-5.00)$ & $3.0(2.56-5.30)$ & 0.191 \\
\hline ər [median (5-95\%)] & $405(220-1016)$ & $290(220-874)$ & 310 (220-1549) & 0.056 \\
\hline nedian (5-95\%)] & $3.06(0.87-7.00)$ & $1.92(0.82-7.00)$ & $2.00(0.97-8.10)$ & 0.011 \\
\hline 3 [median (5-95\%)] & $15.1(5.3-35.0)$ & $12.0(5.0-34.4)$ & $13.2(7.0-46.6)$ & \multirow{3}{*}{$\begin{array}{l}0.011 \\
0.016\end{array}$} \\
\hline $\mathrm{LMR} \leq 5.32$ & $111(34.7)$ & $85(76.6)$ & $26(23.4)$ & \\
\hline $\mathrm{LMR} \leq 5.32$ & 209 (65.3) & $182(87.1)$ & 27 (12.9) & \\
\hline $\mathrm{PMR} \leq 819$ & $101(31.6 \%)$ & $78(77.2 \%)$ & $23(22.8 \%)$ & \multirow{2}{*}{0.042} \\
\hline $\mathrm{PMR}>819$ & $219(68.4 \%)$ & $189(86.3 \%)$ & $30(13.7 \%)$ & \\
\hline \multicolumn{2}{|l|}{ rausal status } & & & \multirow[t]{2}{*}{0.02} \\
\hline Premenopausal & $224(70.0)$ & $194(86.6)$ & $30(13.4)$ & \\
\hline Postmenopausal & $96(30.0)$ & $73(76.0)$ & $23(24.0)$ & \\
\hline tus & $129(403)$ & 114 (884) & $15(116)$ & \multirow[t]{2}{*}{0.051} \\
\hline $\mathrm{ER}>1 \%$ & $191(59.7)$ & $153(80.1)$ & $\begin{array}{l}15(11.0) \\
38(19.9)\end{array}$ & \\
\hline tus & & & & \multirow{3}{*}{0.451} \\
\hline$P R \leq 1 \%$ & $154(48.1)$ & $131(85.1)$ & $23(14.9)$ & \\
\hline PR $\square 1 \%$ & 166 (51.9) & $136(81.9)$ & $30(18.1)$ & \\
\hline \multicolumn{2}{|l|}{ status } & & & \multirow[t]{3}{*}{0.069} \\
\hline Positive & $145(45.3)$ & 127 (87.6) & $18(12.4)$ & \\
\hline Negative & $175(54.7)$ & $140(80.0)$ & $35(20.0)$ & \\
\hline \multicolumn{2}{|l|}{ tatus } & & & \multirow[t]{3}{*}{0.301} \\
\hline Positive & $230(71.9)$ & $195(84.8)$ & $35(15.2)$ & \\
\hline Negative & $90(20.1)$ & $72(80.0)$ & $18(20.0$ & \\
\hline $\begin{array}{l}\text { sgical type } \\
\text { IDC }\end{array}$ & $286(894)$ & $239(836)$ & $47(164)$ & \multirow[t]{2}{*}{0.857} \\
\hline Others & $34(10.6)$ & $28(82.4)$ & $28(82.4)$ & \\
\hline \multirow{2}{*}{ sgical grade } & & & & \multirow[t]{2}{*}{0.748} \\
\hline & 169 & $143(84.6)$ & $26(15.4)$ & \\
\hline III & 151 & $124(82.1)$ & $27(17.9)$ & \multirow{3}{*}{0.002} \\
\hline \multirow[t]{2}{*}{ T1-2 } & $260(81.3)$ & $225(86.5)$ & 35 (13.5) & \\
\hline & $60(18.7)$ & $42(70.0)$ & $18(30.0)$ & \\
\hline \multirow[b]{2}{*}{$\mathrm{N} 2-3$} & $290(90.6)$ & $247(85.2)$ & $43(14.8)$ & \multirow{2}{*}{0.009} \\
\hline & $30(9.4)$ & $20(66.7)$ & $10(33.3)$ & \\
\hline \multirow{7}{*}{$\begin{array}{c}\text { ular subtype } \\
\text { HR+HER2- } \\
\text { HR+HER2+ } \\
\text { HR-HER2+ } \\
\text { HR-HER2- } \\
\end{array}$} & $237(74.1)$ & $200(844)$ & $37(156)$ & \multirow{2}{*}{0.439} \\
\hline & $83(25.9)$ & $67(80.7)$ & $16(19.3)$ & \\
\hline & & & & \multirow[t]{5}{*}{0.058} \\
\hline & $111(34.7)$ & $84(75.7)$ & $27(24.3)$ & \\
\hline & $77(24.1)$ & 67 (87.0) & $10(13.0)$ & \\
\hline & $70(21.8)$ & $62(88.6)$ & $8(11.4)$ & \\
\hline & $62(19.4)$ & $54(87.1)$ & $8(12.9)$ & \\
\hline
\end{tabular}

The age, BMI, fibrinogen, D-Dimer, CEA and CA15-3 $p$ values were determined by Mann-Whitney U tests, while other $\mathrm{p}$ values were determined by Chi square tests

$B M I$ body mass index, $C E A$ carcinoembryonic antigen, $C A 15-3$ cancer antigen 15-3, LMR lymphocyte to monocyte ratio, $P M R$ platelet to monocyte ratio

Table 2. Predictive factors for type 1 regression after NAC in 320 patients, estimated by univariate and multivariate logistic regression analyses 


\begin{tabular}{|c|c|c|c|c|}
\hline \multirow[t]{2}{*}{ Factors } & \multicolumn{2}{|c|}{ Univariate analysis } & \multicolumn{2}{|c|}{ Multivariate analysis } \\
\hline & OR (95\% CI) & $p$ value & OR (95\% CI) & $p$ value \\
\hline Age, years & $0.97(0.94-1)$ & 0.099 & & \\
\hline BMI, kg/m2 & $0.93(0.86-1.01)$ & 0.096 & & \\
\hline Fibrinogen, g/L & $0.72(0.5-1.04)$ & 0.078 & & \\
\hline D-Dimer, ng/mL & $1(1-1)$ & 0.053 & & \\
\hline CEA, ng/mL & $1(0.97-1.06)$ & 0.924 & & \\
\hline $\mathrm{CA} 15-3, \mathrm{U} / \mathrm{ml}$ & $0.98(0.95-1)$ & 0.042 & & \\
\hline LMR & & & $1.99(1.07-3.74)$ & 0.031 \\
\hline LMR $\leq 5.32$ & 1 & & & \\
\hline $\begin{array}{l}\text { LMR } 15.32 \\
\text { PMR }\end{array}$ & $2.06(1.13-3.75)$ & 0.018 & & \\
\hline PMR $\leq 819$ & 1 & & & \\
\hline PMR $\square 819$ & $1.86(1.02-3.40)$ & 0.044 & & \\
\hline Menopausal status & & & $0.50(0.26-0.96)$ & 0.037 \\
\hline Premenopausal & 1 & & & \\
\hline Postmenopausal & $0.49(0.27-0.91)$ & 0.021 & & \\
\hline ER status & & & $0.45(0.23-0.90)$ & 0.024 \\
\hline $\mathrm{ER} \leq 1 \%$ & 1 & & & \\
\hline ER $>1 \%$ & $0.53(0.27-0.91)$ & 0.054 & & \\
\hline PR status & & & & \\
\hline $\mathrm{PR} \leq 1 \%$ & 1 & & & \\
\hline $\mathrm{PR}>1 \%$ & $0.8(0.44-1.44)$ & 0.451 & & \\
\hline HER2 status & & & & \\
\hline Positive & 1 & & & \\
\hline Negative & $0.57(0.31-1.05)$ & 0.071 & & \\
\hline $\begin{array}{l}\text { Ki67 status } \\
\text { Positive }\end{array}$ & 1 & & & \\
\hline Negative & 0.72ロ0.38-1.35ロ & 0.302 & & \\
\hline $\begin{array}{c}\text { Histological type } \\
\text { IDC }\end{array}$ & & & & \\
\hline $\begin{array}{c}\text { IDC } \\
\text { Others }\end{array}$ & $\frac{1}{2}$ & 0957 & & \\
\hline Histological grade & & ו & & \\
\hline I & 1 & & & \\
\hline II & $0.89(0.10-7.14)$ & 0.918 & & \\
\hline III & $0.81(0.09-7.06)$ & 0.851 & & \\
\hline T stage & 1 & & $0.38(0.19-0.76)$ & 0.006 \\
\hline $\begin{array}{l}11-2 \\
\text { T3-4 }\end{array}$ & $0.36(0.19-0.71)$ & 0.003 & & \\
\hline N stage & & & $0.38(0.16-0.93)$ & 0.034 \\
\hline $\begin{array}{l}\text { N0-1 } \\
\text { N2-3 }\end{array}$ & $\frac{1}{1}$ & 0012 & & \\
\hline $\mathrm{N} 2-3$ & 0.35 & & & \\
\hline
\end{tabular}

Multivariate analysis was based on forward stepwise logistic regression (likelihood ratio) analysis.

Bold $p$ values in the univariate analysis indicate statistical significance $(p \leq 0.05)$ or borderline significant. All variables with $p$ values in bold were incorporated into the multivariate analysis.

OR odds ratio, CI confidence interval, BMI body mass index, CEA carcinoembryonic antigen, CA15-3 cancer antigen 15-3, $L M R$ lymphocyte to monocyte ratio, $P M R$ platelet to monocyte ratio

A nomogram was established based on menopausal status, ER status, T stage, $\mathrm{N}$ stage and LMR to predict the tumor regression pattern after NAC (Figure 2). To calculate the likelihood of type 1 regression, each point for the five independent factors was added and yielded a final score. A virtual perpendicular line can be drawn between the total points bar and the risk bar at the bottom of the nomogram. Each final score corresponds to a single risk of type 1 regression. The AUC of the established nomogram was 0.70 (95\% Cl 0.62-0.78). The calibration plot accompanied by the Hosmer-Lemeshow goodness-of-fit (GOF) test $(p$-value $=0.9)$ showed good consistency between the estimated probabilities and the actual rate of type 1 cases. The decision curve employed showed that adopting our nomogram would bring improved clinical outcome. 


\section{Discussion}

Our study established a nomogram based on baseline characteristics to predict tumor regression patterns after NAC in breast cancer. Five clinicopathological factors and laboratory indexes, including T stage, $N$ stage, menopausal status, ER status, and LMR, were included in the nomogram. Patients who were premenopausal or ER-negative and had higher LMR and later T/N stages had a higher probability of type 1 tumor regression. Such patients had a relatively lower probability of type 2 regression, which means they are not favored candidates for BCS. Consistent with the findings of previous literature[13,14], large tumor size, later nodal stage and lower ER expression are associated with a nonconcentric regression of the tumor after NAC.

Tumor regression patterns have been explored by various institutes. Molecular subtypes have been utilized to correlate with tumor regression patterns, but no consensus has been reached[15,16]. In our study, HR+/HER2- breast cancers had a significantly higher rate of type 2 regression (24.3\%), which could be explained by their lower aggressiveness in nature and lower sensitivity to chemotherapy. HR-/HER2+ and HR-/HER2- breast cancers were found to have a better response to presurgical chemotherapy, especially in HR-HER2+ cases when HER2-targeted therapy was added[17]. In the present study, the rates of type 2 regression in HR-HER2+ and HR-HER2- cases were only $11.4 \%$ and $12.9 \%$, respectively. Similarly, a meta-analysis revealed that different breast cancer subtypes showed different responses to NAC[18]. Molecular subtypes could, therefore, be considered a predictor for tumor regression patterns. However, it was not incorporated into our nomogram, considering that it is decided by ER/PR/HER2 status and Ki67 index and might cause confounding bias if they were integrated together. In our study, the BCS rate was $38.2 \%(102 / 267)$ and $13.2 \%$ (7/53) in patients with type 1 and type 2 regression, respectively. For patients who desire BCS, a consultation with their surgeons immediately before the initial preoperative systemic treatment based on the predicted result of the nomogram would be helpful.

Presurgical breast MRI is the favored modality for evaluating residual cancer after NAC[19,20]. Due to its high sensitivity and low specificity, determining the tumor regression pattern by MRI may not be adequately accurate[21,22]. Furthermore, the concordance rate between MRI and pathology results was barely satisfactory[23,24]. Plana et al. reported that presurgical MRI detected additional breast diseases and prompted conversion from local excision to more extensive surgery in $12.8 \%$ of women and nearly half of these were inappropriate due to the incidence of false positive[25]. Thus, the tumor regression patterns are determined by pathology results. The presurgical breast MRI does not provide information on the possible tumor regression model at the early phase. Our nomogram utilized the baseline characteristics of breast tumors, which allows the early prediction of tumor response to NAC, and the treatment strategy may be modified in the early stage.

Laboratory indexes, including peripheral immune cell counts and nutritional indicators, are inexpensive and easily accessible. In our study, 4 laboratory indexes, including LMR, PLR, fibrinogen and D-dimer, were explored. Higher levels of D-dimer and fibrinogen are associated with advanced malignancy in breast cancers, and these parameters are linked to homeostasis and tumor progression. PLR has been 
recognized as a predictor for chemotherapy response and a prognostic factor for better DFS outcomes $[26,27]$. LMR has been studied in various types of cancers, especially in alimentary system tumors[26,28]. Our study also identified LMR as an independent factor for predicting tumor regression patterns. A higher LMR was associated with a higher probability of type 1 regression, which could be explained by the stronger aggressiveness in tumors with a higher LMR[29,30] and increased sensitivity to chemotherapy. LMR can be recognized as a promising predictive factor for the tumor response to NAC. To the best of our knowledge, no other studies have reported the correlation between LMR and tumor regression patterns.

Our study had a retrospective design, and we could only incorporate accessible factors in clinical practice. Other variables, e.g., from gene levels or unknown aspects, may need further exploration. The AUC of our model needs to be improved possibly by incorporating other types of variables. A radiomic signature combining multiparametric MRI features would be promising. Meanwhile, type 1 regression is encountered much more frequently than type 2 regression in clinical observation, which leads to an imbalance in the number of cases with the two regression patterns. This situation is consistent with our clinical practice since most tumors have a good response to chemotherapy (and HER2-targeted therapy, e.g., trastuzumab in HER2-positive cases). Additionally, the nomogram established based on single-center data needs further external validation in other cohorts.

In conclusion, HR+/HER2- breast cancers are more likely to have type 2 regression after neoadjuvant chemotherapy. Five baseline factors, including clinicopathological factors and laboratory indexes, were incorporated to establish a nomogram, which exhibited a sufficient discriminatory ability for predicting different patterns of tumor regression.

\section{Abbreviations}

Neoadjuvant Chemotherapy (NAC)

Pathologic Complete Response (pCR)

Goodness-Of-Fit (GOF)

Ipsilateral Breast Tumor Recurrence rate (IBTR)

Lymphocyte to Monocyte Ratio (LMR)

Platelet to Monocyte Ratio (PMR)

Hormone Receptor-positive, HER2-negative (HR+/HER2-)

\section{Declarations}

\section{Acknowledgments}


This study was funded by the National Natural Science Foundation of China (81871513), Natural Science Foundation of Guangdong Province, China (2017A030313882) and CSCO-Constant Rui Tumor Research Fund, China (Y-HR2016-067).

\section{Ethics approval and consent to participate}

All procedures performed in this study, which involved human participants, were in accordance with the ethical standards of the institutional and/or nation research committee and with the 1964 Helsinki Declaration and its later amendments or comparable ethical standards. For this type of study formal consent is not required.

\section{Consent for publication}

Not applicable.

\section{Availability of data and material}

Original data are available from our corresponding author upon reasonable request.

\section{Competing interests}

The authors declare that they have no conflict of interest.

\section{Funding}

This study was funded by the National Natural Science Foundation of China (81871513), Natural Science Foundation of Guangdong Province, China (2017A030313882) and CSCO-Constant Rui Tumor Research Fund, China (Y-HR2016-067).

\section{Authors' contributions}

Xiaosheng Zhuang: Conceptualization; Data curation; Formal analysis; Investigation; Methodology; Software; Writing - original draft; Writing - review \& editing.

Minyi Cheng: Conceptualization; Formal analysis; Investigation; Methodology; Software; Writing - original draft; Writing - review \& editing.

Liulu Zhang: Conceptualization; Formal analysis; Investigation; Methodology;

Fei Ji: Data curation; Investigation; Writing - review \& editing.

Teng Zhu: Data curation; Writing - review \& editing.

Ciqiu Yang: Data curation; Software; Investigation.

Mei Yang: Data curation; Investigation. 
Hongfei Gao: Methodology; Software; Writing - review \& editing.

Jieqing Li: Methodology; Writing - review \& editing.

Kun Wang: Conceptualization; Funding acquisition; Resources; Supervision.

\section{References}

1. Kaufmann M, Hortobagyi GN, Goldhirsch A, Scholl S, Makris A, Valagussa P, et al. Recommendations from an international expert panel on the use of neoadjuvant (primary) systemic treatment of operable breast cancer: an update. Journal of Clinical Oncology 2006;24:1940-9.

2. Thompson A, Moulder-Thompson S. Neoadjuvant treatment of breast cancer. annals of Oncology 2012;23:x231-x6.

3. Derks MG, van de Velde CJ. Neoadjuvant chemotherapy in breast cancer: more than just downsizing. The Lancet Oncology 2018;19:2-3.

4. Fukada I, Araki K, Kobayashi K, Shibayama T, Takahashi S, Gomi N, et al. Pattern of tumor shrinkage during neoadjuvant chemotherapy is associated with prognosis in low-grade luminal early breast cancer. Radiology 2018;286:49-57.

5. Straver ME, Loo CE, Rutgers EJ, Oldenburg HS, Wesseling J, Peeters M-JTV, et al. MRI-model to guide the surgical treatment in breast cancer patients after neoadjuvant chemotherapy. Annals of surgery 2010;251:701-7.

6. Ling DC, Sutera PA, larrobino NA, Diego EJ, Soran A, Johnson RR, et al. Is Multifocal Regression a Risk Factor for Ipsilateral Breast Tumor Recurrence in the Modern Era After Neoadjuvant Chemotherapy and Breast Conservation Therapy? International Journal of Radiation Oncology* Biology* Physics 2019;104:869-76.

7. Asselain B, Barlow W, Bartlett J, Bergh J, Bergsten-Nordström E, Bliss J, et al. Long-term outcomes for neoadjuvant versus adjuvant chemotherapy in early breast cancer: meta-analysis of individual patient data from ten randomised trials. The Lancet Oncology 2018;19:27-39.

8. Liu Z, Li Z, Qu J, Zhang R, Zhou X, Li L, et al. Radiomics of Multiparametric MRI for Pretreatment Prediction of Pathologic Complete Response to Neoadjuvant Chemotherapy in Breast Cancer: A Multicenter Study. Clin Cancer Res 2019;25:3538-47.

9. F Z, M H, H Z, K C, J J, Y W, et al. A Nomogram to Predict the Pathologic Complete Response of Neoadjuvant Chemotherapy in Triple-Negative Breast Cancer Based on Simple Laboratory Indicators. Annals of surgical oncology 2019;26:3912-9.

10. Gradishar WJ, Anderson BO, Balassanian R, Blair SL, Burstein HJ, Cyr A, et al. Breast cancer, version 4.2017, NCCN clinical practice guidelines in oncology. Journal of the National Comprehensive Cancer Network 2018;16:310-20.

11. Poggio F, Bruzzone M, Ceppi M, Pondé N, La Valle G, Del Mastro L, et al. Platinum-based neoadjuvant chemotherapy in triple-negative breast cancer: a systematic review and meta-analysis. Annals of 
oncology 2018;29:1497-508.

12. von Minckwitz G, Untch M, Blohmer JU, Costa SD, Eidtmann H, Fasching PA, et al. Definition and impact of pathologic complete response on prognosis after neoadjuvant chemotherapy in various intrinsic breast cancer subtypes. J Clin Oncol 2012;30:1796-804.

13. Wang S, Zhang Y, Yang X, Fan L, Qi X, Chen Q, et al. Shrink pattern of breast cancer after neoadjuvant chemotherapy and its correlation with clinical pathological factors. World journal of surgical oncology 2013;11:166.

14. Raphael J, Gandhi S, Li N, Lu F-I, Trudeau M. The role of quantitative estrogen receptor status in predicting tumor response at surgery in breast cancer patients treated with neoadjuvant chemotherapy. Breast cancer research and treatment 2017;164:285-94.

15. Loo CE, Straver ME, Rodenhuis S, Muller SH, Wesseling J, Vrancken Peeters M-JT, et al. Magnetic resonance imaging response monitoring of breast cancer during neoadjuvant chemotherapy: relevance of breast cancer subtype. Journal of Clinical Oncology 2011;29:660-6.

16. Li M, Xu B, Shao Y, Liu H, Du B, Yuan J. Magnetic resonance imaging patterns of tumor regression in breast cancer patients after neo-adjuvant chemotherapy, and an analysis of the influencing factors. The breast journal 2017;23:656-62.

17. Gianni L, Pienkowski T, Im YH, Roman L, Tseng LM, Liu MC, et al. Efficacy and safety of neoadjuvant pertuzumab and trastuzumab in women with locally advanced, inflammatory, or early HER2-positive breast cancer (NeoSphere): a randomised multicentre, open-label, phase 2 trial. Lancet Oncol 2012;13:25-32.

18. Houssami N, Macaskill P, von Minckwitz G, Marinovich ML, Mamounas E. Meta-analysis of the association of breast cancer subtype and pathologic complete response to neoadjuvant chemotherapy. Eur J Cancer 2012;48:3342-54.

19. Kim SY, Cho N, Park IA, Kwon BR, Shin SU, Kim SY, et al. Dynamic Contrast-enhanced Breast MRI for Evaluating Residual Tumor Size after Neoadjuvant Chemotherapy. Radiology 2018;289:327-34.

20. Ko BS, Kim N, Lee JW, Kim HJ, Chung IY, Kim J, et al. MRI-based 3D-printed surgical guides for breast cancer patients who received neoadjuvant chemotherapy. Sci Rep 2019;9:11991.

21. Houssami N, Ciatto S, Macaskill P, Lord SJ, Warren RM, Dixon JM, et al. Accuracy and surgical impact of magnetic resonance imaging in breast cancer staging: systematic review and metaanalysis in detection of multifocal and multicentric cancer. J Clin Oncol 2008;26:3248-58.

22. Marinovich ML, Houssami N, Macaskill P, Sardanelli F, Irwig L, Mamounas EP, et al. Meta-analysis of magnetic resonance imaging in detecting residual breast cancer after neoadjuvant therapy. J Natl Cancer Inst 2013;105:321-33.

23. Marinovich ML, Macaskill P, Irwig L, Sardanelli F, von Minckwitz G, Mamounas E, et al. Meta-analysis of agreement between MRI and pathologic breast tumour size after neoadjuvant chemotherapy. $\mathrm{Br} \mathrm{J}$ Cancer 2013;109:1528-36.

24. Choi WJ, Kim HH, Cha JH, Shin HJ, Chae EY, Yoon GY. Complete response on MR imaging after neoadjuvant chemotherapy in breast cancer patients: Factors of radiologic-pathologic discordance. 
Eur J Radiol 2019;118:114-21.

25. Plana MN, Carreira C, Muriel A, Chiva M, Abraira V, Emparanza JI, et al. Magnetic resonance imaging in the preoperative assessment of patients with primary breast cancer: systematic review of diagnostic accuracy and meta-analysis. Eur Radiol 2012;22:26-38.

26. De Giorgi U, Mego M, Scarpi E, Giordano A, Giuliano M, Valero V, et al. Association between circulating tumor cells and peripheral blood monocytes in metastatic breast cancer. Ther Adv Med Oncol 2019;11:1758835919866065.

27. Hu Y, Wang S, Ding N, Li N, Huang J, Xiao Z. Platelet/Lymphocyte Ratio Is Superior to Neutrophil/Lymphocyte Ratio as a Predictor of Chemotherapy Response and Disease-free Survival in Luminal B-like (HER2(-)) Breast Cancer. Clin Breast Cancer 2020.

28. Miyamoto Y, Hiyoshi Y, Daitoku N, Okadome K, Sakamoto Y, Yamashita K, et al. Naples Prognostic Score Is a Useful Prognostic Marker in Patients With Metastatic Colorectal Cancer. Dis Colon Rectum 2019;62:1485-93.

29. Nishijima TF, Muss HB, Shachar SS, Tamura K, Takamatsu Y. Prognostic value of lymphocyte-tomonocyte ratio in patients with solid tumors: A systematic review and meta-analysis. Cancer Treat Rev 2015;41:971-8.

30. Hu RJ, Liu Q, Ma JY, Zhou J, Liu G. Preoperative lymphocyte-to-monocyte ratio predicts breast cancer outcome: A meta-analysis. Clin Chim Acta 2018;484:1-6.

\section{Figures}

A

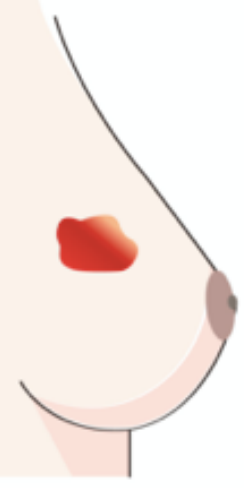

D

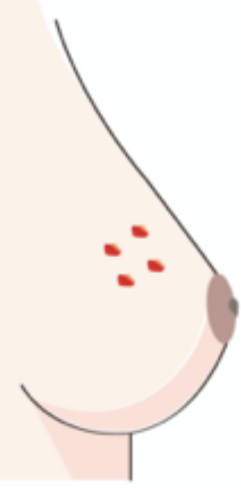

E

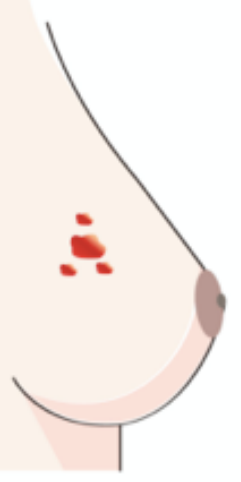

B

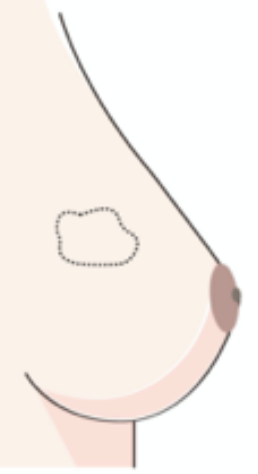

C

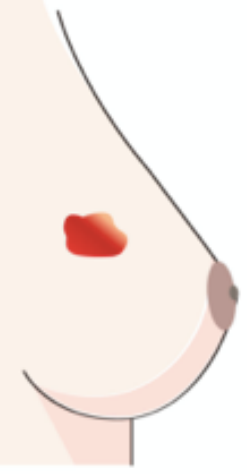

F

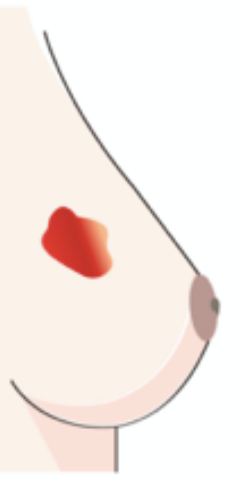

G

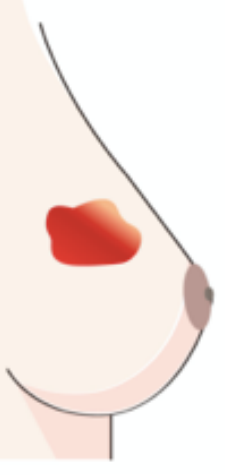


Figure 1

Tumor regression patterns after neoadjuvant chemotherapy A. Primary cancer B. Type 1 regression (complete remission) C. Type 1 regression (concentric regression) D. Type 2 regression (multiple residual foci) E. Type 2 regression (main residual disease with satellite foci) F. Type 2 regression (stable disease) G. Type 2 regression (progressive disease)

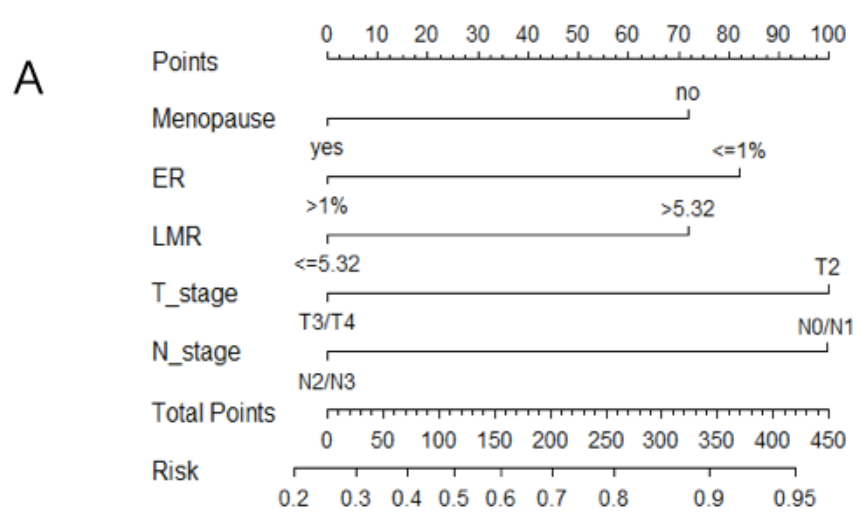

B

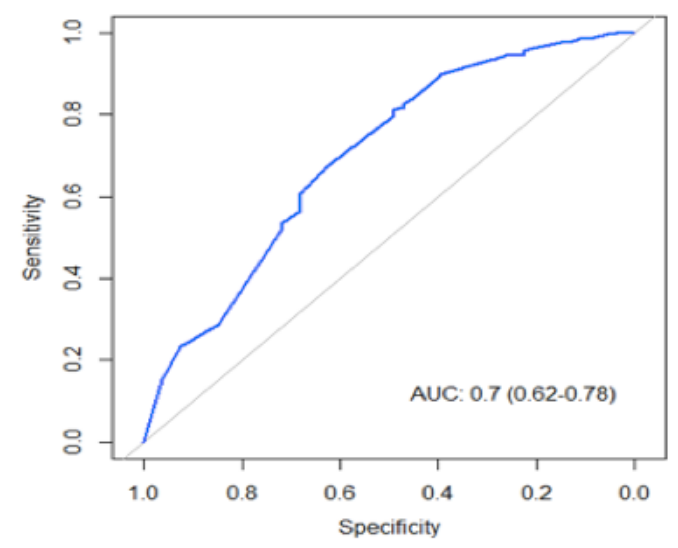

C
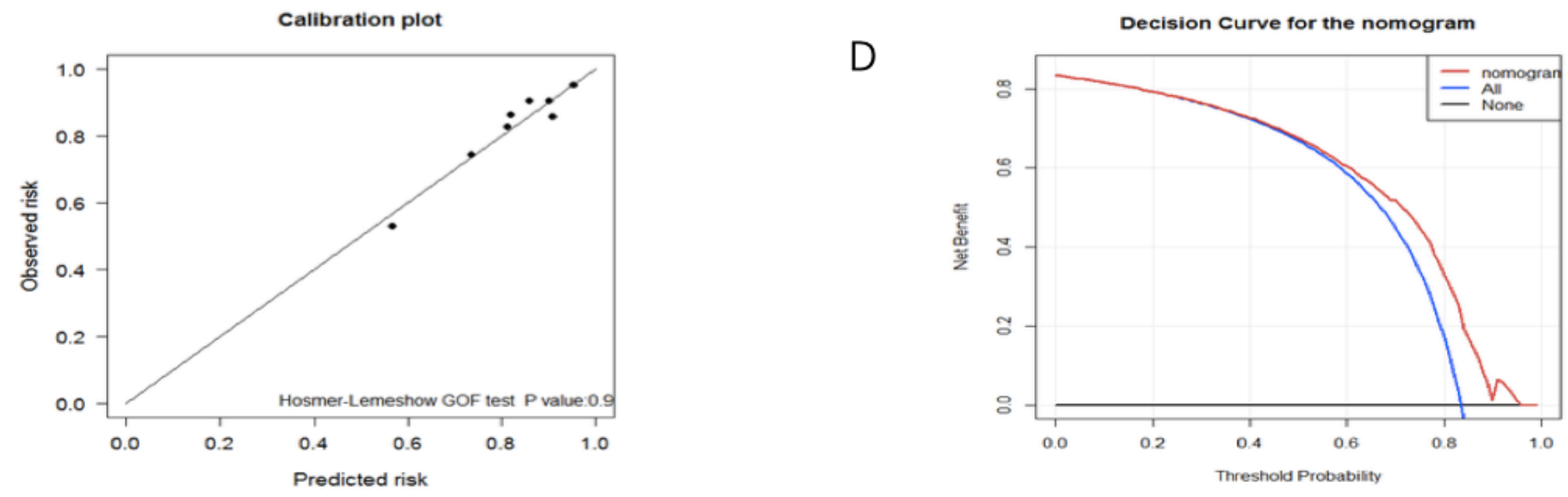

\section{Figure 2}

The nomogram and its decision curve and calibration plot. A. The nomogram for predicting type 1 regression after NAC in breast cancer patients. B. The nomogram established yielded an AUC of 0.7 (0.62$0.78)$, demonstrating its discrminatory ability to predict tumor regression patterns after NAC. C. The calibration plot accompanied by the Hosmer-Lemeshow goodness-of-fit (GOF) test ( $p$ value $=0.9$ ) shows good consistency between the estimated probability and the actual rate of type 1 cases, of which $p$ values $>0.05$ indicated a good fit of the model test. D. The decision curve of the nomogram. The $y$ axis measures the net benefit. The red line delineates the nomogram. The blue line represents the assumption that all patients showed type 1 regression. The black line represents the assumption that no patients showed type 1 regression. 